\title{
Integer Semigroups Associated with Dumont-Thomas Numeration Systems
}

\author{
Víctor F. Sirvent \\ Departamento de Matemáticas, Universidad Simón Bolívar, Apartado 89000, Caracas 1086, Venezuela \\ Correspondence should be addressed to Víctor F. Sirvent; vsirvent@usb.ve
}

Received 30 September 2013; Accepted 13 November 2013; Published 23 January 2014

Academic Editors: C. da Fonseca, A. Glen, P. E. Jorgensen, A. V. Kelarev, and C.-K. Lin

Copyright (C) 2014 Víctor F. Sirvent. This is an open access article distributed under the Creative Commons Attribution License, which permits unrestricted use, distribution, and reproduction in any medium, provided the original work is properly cited.

\begin{abstract}
Given a primitive substitution, we define different binary operations on infinite subsets of the nonnegative integers. These binary operations are defined with the help of the Dumont-Thomas numeration system; that is, a numeration system associated with the substitution. We give conditions for these semigroups to have an identity element. We show that they are not finitely generated. These semigroups define actions on the set of positive integers. We describe the orbits of these actions. We also estimate the density of these sets as subsets of the positive integers.
\end{abstract}

\section{Introduction}

Integer semigroups have been studied in different contexts (cf. [1-4]). One of the well known binary operations is the Fibonacci multiplication, introduced by Knuth [1]. This operation is related to the dynamical system obtained by the Fibonacci substitution (cf. [3]). It has been generalized in different ways to the tribonacci substitution; see [4-6]. The author studied the usage of binary operations on the set of nonnegative integers, in order to describe the self-similar structure of the $k$-bonacci substitutions [4] and on the socalled flipped tribonacci substitution [7].

In the present article, we introduce binary operations on infinite subsets of the nonnegative integers so that they are integer semigroups. These binary operations are associated with a substitution, in particular to the numeration system defined by the substitution, the so-called Dumont-Thomas numeration system. These numeration systems play an important role in the study of Rauzy fractals; for details see $[8,9]$ and references within. The integer semigroups defined in the paper, in general, do not have identity elements. We explore when they have identity elements. In Theorem 3, we show that these semigroups are not finitely generated.

Moreover, we show that these semigroups define actions on the set of positive integers, and we describe the orbits of these actions.
In Section 4, we study the density of these semigroups as subsets of the positive integers.

\section{Substitutions and Automata}

A substitution on a finite alphabet $\mathscr{A}=\{1, \ldots, k\}$ is a map $\zeta$ from $\mathscr{A}$ to the set of finite words on $\mathscr{A}$; that is, $\mathscr{A}^{*}=\mathrm{U}_{i \geq 0} \mathscr{A}^{i}$. This map extends to $\mathscr{A}^{*}$ by concatenation by $\zeta(\emptyset)=\emptyset$ and $\zeta(U V)=\zeta(U) \zeta(V)$, for all $U, V \in \mathscr{A}^{*}$. Let $A^{\mathbb{N}}$ denote the set of one-sided infinite sequences in $\mathscr{A}$. The map $\zeta$ is extended to $\mathscr{A}^{\mathbb{N}}$ in the obvious way. We call $\mathbf{u} \in \mathscr{A}^{\mathbb{N}}$ a fixed point of $\zeta$ if $\zeta(\mathbf{u})=\mathbf{u}$ and periodic if there exists $l>0$ so that it is fixed for $\zeta^{l}$. There is a dynamical system associate to the sequence $\mathbf{u}$, called substitution dynamical system; for details see [8-10].

The incidence matrix of the substitution $\zeta$ is defined as the matrix $M=\left(M_{i j}\right)$ whose entry $M_{i j}$ is the number of occurrences of the symbol $j$ in the word $\zeta(i)$, for $1 \leq i, j \leq k$. We say the substitution is primitive if its incidence matrix is primitive; that is, all the entries of $M^{l}$ are positive for some $l>0$. We shall assume throughout the paper that the substitutions are primitive.

For a primitive substitution there are a finite number of periodic points. So, we can assume without loss of generality that a primitive substitution has a fixed point; say $\mathbf{u}=u_{1} u_{2} \ldots$ and $u_{1}=1$; that is, the first symbol of $\zeta(1)$ is 1 . 


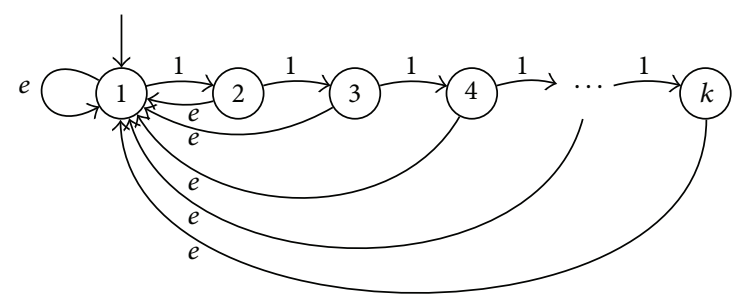

Figure 1: The $k$-bonacci automaton.

Let $W \subset \mathscr{A}^{*}=\cup_{i \geq 0} \mathscr{A}^{i}$ be a set of finite words on the alphabet $\mathscr{A}=\{1, \ldots, k\}$. An automaton over $\mathscr{A}, \mathfrak{A}=(Q, W$, $E, I)$ is a direct graph labelled by elements of $\mathscr{A}$. $Q$ is the set of states, $I \subset Q$ is the set of initial states, $W$ is the set of labels, and $E \subset Q \times W \times Q$ is the set of labelled edges or transitions. If $(p, w, q) \in E$, we say that $w$ is a transition between $p$ and $q$.

The prefix automaton of the substitution $\zeta$ (cf. [11]) is the automaton $\mathfrak{A}=(Q, W, E, I)$ so that one has the following.

(i) $\mathrm{Q}=\mathscr{A}$.

(ii) $W=$ Pref, the set of proper prefixes of the words $\zeta(p)$, for $p \in \mathscr{A}$. We will denote by $e$ the empty prefix.

(iii) $(p, w, q)$ is in $E$ if $p, q$ are elements of $\mathscr{A}, w \in W$ and $w q$ is a prefix of $\zeta(p)$.

(iv) $I=\{1\}$.

In Figure 1, it is shown the prefix automaton of the $k$ bonacci substitution; that is, $\zeta(1)=12, \zeta(2)=13, \ldots, \zeta(k-$ $1)=1 k$, and $\zeta(k)=1$.

The automaton reads words from left to right. A finite path in the automaton, $\mathfrak{A}$, is word in $E$, the set of transitions:

$$
\left(p_{m}, a_{m}, q_{m}\right)\left(p_{m-1}, a_{m-1}, q_{m-1}\right) \cdots\left(p_{0}, a_{0}, q_{0}\right)
$$

such that $q_{i}=p_{i-1}$ for $1 \leq i \leq m$ and $p_{m} \in I$; however, we usually denote the paths using only the labels, that is, $a_{m} \cdots a_{0}$. We say that the path $a_{m} \cdots a_{0}$ terminates at the state $q_{0}$ and we say that the path passes through the stateq, if there is $i \in$ $\{0, \ldots, m\}$ such that $p_{i}=q$, in (1). See [12] for more details on automata theory.

Theorem 1 (see [11, 13]). Let $U$ be a nonempty prefix of $\mathbf{u}$, the fixed point of the substitution $\zeta$. Then there exists a unique path on the prefix automaton of $\zeta: a_{n} a_{n-1} \cdots a_{0}$ such that $a_{n} \neq e$ and $U=\zeta^{n}\left(a_{n}\right) \zeta^{n-1}\left(a_{n-1}\right) \cdots \zeta\left(a_{1}\right) a_{0}$. Conversely, to any such path, there corresponds a prefix of $\mathbf{u}$, given by the above formula.

Due to this theorem, we can associate numeration systems for the positive integers, in the following way. Let $n$ be a positive integer and $U$ the prefix of length $n$ of $\mathbf{u}$; we define $\epsilon: \mathbb{N} \rightarrow W^{*}$, by $\epsilon(n)=a_{l} a_{l-1} \cdots a_{1} a_{0}$, where $a_{l} \neq e$ and $U=\zeta^{l}\left(a_{l}\right) \zeta^{l-1}\left(a_{l-1}\right) \cdots \zeta\left(a_{1}\right) a_{0}$. Moreover, this representation is unique. This numeration system is called the DumontThomas numeration system associated with the substitution $\zeta$ (cf. $[13,14])$. For general theory of numeration systems see $[8,14-18]$.

\section{Semigroups}

We consider the following subset of positive integers:

$$
\begin{aligned}
\mathscr{P}:=\left\{n \in \mathbb{N}: \epsilon(n)=a_{l} \cdots a_{0}, \text { such that } a_{l} \cdots a_{0}\right. \text { is path in } \\
\mathfrak{\mathcal { H }} \text { terminating at state } 1\} .
\end{aligned}
$$

We define " $\star$ ", a binary operation in $\mathscr{P}$, in the following way: let $n, m \in \mathscr{P}$ such that $\epsilon(n)=a_{l} a_{l-1} \cdots a_{1} a_{0}$ and $\epsilon(m)=$ $b_{r} b_{r-1} \cdots b_{0}$, so

$$
\epsilon(n \star m):=a_{l} a_{l-1} \cdots a_{1} a_{0} b_{r} b_{r-1} \cdots b_{0} .
$$

This binary operation is well defined since the paths in the prefix-automaton, associated with the elements of $\mathscr{P}$, terminate at the initial state, so we can concatenate them. It is clear that the operation $\star$ is associative. Hence, $(\mathscr{P}, \star)$ is a semigroup.

However, the binary operation $\star$ is not always commutative; for example, in the Fibonacci case, we consider $\epsilon(2)=1 e$ and $\epsilon(3)=1 e e$, so $\epsilon(2 \star 3)=1 e 1 e e=\epsilon(10)$ and $\epsilon(3 \star 2)=$ $1 e e 1 e=\epsilon(9)$, so $2 \star 3=10$ and $3 \star 2=9$.

If the word $\zeta(1)$ starts with the symbol 1 , then in the prefix automation the transition $(1, e, 1)$ is allowed, so $e \ldots$ $e a_{l} a_{l-1} \cdots a_{1} a_{0}$ is a path in $\mathfrak{A}$, and

$$
\begin{aligned}
& \zeta^{l}\left(a_{l}\right) \zeta^{l-1}\left(a_{l-1}\right) \cdots \zeta\left(a_{1}\right) a_{0} \\
& \quad=\zeta^{l+i}(e) \cdots \zeta^{l+1}(e) \zeta^{l}\left(a_{l}\right) \zeta^{l-1}\left(a_{l-1}\right) \cdots \zeta\left(a_{1}\right) a_{0} .
\end{aligned}
$$

By convention we say that $\epsilon(0)=e$ and let $\mathscr{P}^{\prime}=\mathscr{P} \cup\{0\}$. Clearly $0 \star n=n$, but $n \star 0 \neq n$; since $\zeta^{l}\left(a_{l}\right) \zeta^{l-1}\left(a_{l-1}\right) \ldots \zeta\left(a_{1}\right) a_{0}$, it does not have to be $\zeta^{l+1}\left(a_{1}\right) \zeta^{l}\left(a_{l-1}\right) \cdots \zeta^{2}\left(a_{1}\right) \zeta\left(a_{0}\right)$. Hence, the pair $\left(\mathscr{P}^{\prime}, \star\right)$ has a left identity. If $n \neq 0$ and $\epsilon(n)=$ $a_{l} a_{l-1} \cdots a_{1} a_{0}$, we always consider that $a_{l} \neq e$. However $\left(\mathscr{P}^{\prime}, \star\right)$ is not a semigroup; consider $\epsilon(n \star(e \star m))=a_{l} \cdots a_{0} b_{r} \cdots b_{0}$; since $e \star m=m$ and on the other hand $\epsilon((n \star e) \star m)=$ $a_{l} \cdots a_{0} e b_{r} \cdots b_{0}$, therefore $n \star(e \star m) \neq(n \star e) \star m$. The algebraic structure of the pair $\left(\mathscr{P}^{\prime}, \star\right)$ is an unital, a set with a closed binary operation with identity; see [19].

We shall change the definition of the binary operation so that we have a semigroup with identity. Without loss of generality, we can suppose that the substitution $\zeta$ is of the form $\zeta(1)=1 w 2 w^{\prime}$, where $w^{\prime}$ is a finite word on the alphabet $\mathscr{A}$ and $w=e$ or $w=1 \cdots 1$; that is, $w$ is the empty word or a word consisting only of a concatenation of the symbol 1. Let $\mathscr{P}^{+}$be the set of natural numbers $n$ whose representation is $\epsilon(n)=a_{l} \cdots a_{1} a_{0}$, where the $a_{l} \cdots a_{0}$ is a path in $\mathfrak{A}$ that starts in the initial state and terminates in the state 2 and the path $a_{l} \cdots a_{1}$ terminates at the initial state.

We can think of the set $\mathscr{P}^{+}$as the set of numbers whose representation is a path in the prefix-automaton that starts in the state 1 and terminates in the state 2, such that it passes through 1 in the step before terminating.

We define a binary operation " $\diamond$ " on $\mathscr{P}^{+}$as follows. Let $n, m \in \mathscr{P}^{+}$, whose representations are $\epsilon(n)=a_{l} \cdots a_{1} a_{0}$ and $\epsilon(m)=b_{r} \cdots b_{1} b_{0}$; so

$$
\epsilon(n \diamond m):=a_{l} a_{l-1} \cdots a_{1} b_{r} \cdots b_{0} .
$$




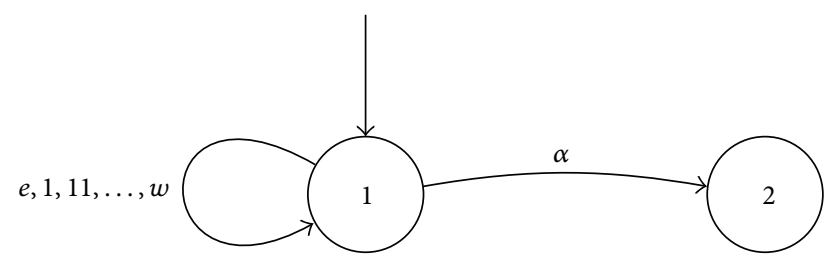

Figure 2: Part of the automaton described in the proof of Proposition 2.

This binary operation is well defined since it is an element of $\mathscr{P}^{+}$. The operation $\diamond$ is associative. Let $n, m, t \in \mathscr{P}^{+}$such that $\epsilon(n)=a_{l} \cdots a_{1} a_{0}, \epsilon(m)=b_{r} \cdots b_{1} b_{0}$ and $\epsilon(t)=c_{s} \cdots c_{1} c_{0}$; so

$$
\begin{aligned}
\epsilon(n \diamond(m \diamond t)) & =\left(a_{l} \cdots a_{1} a_{0}\right) \diamond\left(b_{r} \cdots b_{1} c_{s} \cdots c_{0}\right) \\
& =a_{l} \cdots a_{1} b_{r} \cdots b_{1} c_{s} \cdots c_{0}, \\
\epsilon((n \diamond m) \diamond t) & =\left(a_{l} \cdots a_{1} b_{r} \cdots b_{1} b_{0}\right) \diamond\left(c_{s} \cdots c_{0}\right) \\
& =a_{l} \cdots a_{1} b_{r} \cdots b_{1} c_{s} \cdots c_{0} .
\end{aligned}
$$

The semigroup $\left(\mathscr{P}^{+}, \diamond\right)$ has a left identity; however, there might not be more than one left identity element. Let $(1, \beta, 2)$ be any transition in the prefix automaton $\mathfrak{A}$ from the state 1 to the state 2 , and $\kappa \in \mathbb{N}$ such that $\epsilon(\kappa)=\beta$. By definition $\kappa \in \mathscr{P}^{+}$and $\kappa \diamond n=n$, for any $n \in \mathscr{P}^{+}$. In general $\left(\mathscr{P}^{+}, \diamond\right)$ does not have a right identity.

Proposition 2. Let $\zeta$ be a primitive substitution on $\mathscr{A}$ such that $\zeta(1)=1 w 2 w^{\prime}$ where $w=1 \cdots 1$ or $w=e$ and $w^{\prime}$ is a finite word on the alphabet. If the symbol 2 does not belong to the word $w^{\prime}$, then the identity of the semigroup $\left(\mathscr{P}^{+}, \diamond\right)$ is the element เ of $\mathscr{P}^{+}$whose representation is $\epsilon(\iota)=\alpha$, with $\alpha=1 w$ if $w \neq e$, or $\alpha=1$, if $w=e$.

Proof. Let $\alpha=1 w$ if $w \neq e$, or $\alpha=1$, if $w=e$. We remark that $\alpha$ is a proper prefix of $\zeta(1)$, so $\alpha \in$ Pref, and $(1, \alpha, 2) \in E$ in the prefix automaton; that is, $\alpha$ is a transition from the state 1 to the state 2 . Since $w^{\prime}$ does not have the symbol 2, $\alpha$ is the only transition in the prefix automaton from the state 1 to 2 . The transition in the automaton $\mathfrak{A}$ from the initial state to itself and from the state 1 to the state 2 are shown in Figure 2; in this figure we suppose that the word $w^{\prime}$ does not contain the symbol 1 .

Let $n \in \mathscr{P}^{+}$with $\epsilon(n)=b_{l} \cdots b_{1} b_{0}$. We have $\epsilon(n \diamond \iota)=$ $b_{l} \cdots b_{1} \alpha$. Since $n \in \mathscr{P}^{+}, b_{0}$ is a transition from the state 1 to 2 and there is only one transition from state 1 to state 2 , so $b_{0}=\alpha$. Hence, $n \diamond \iota=n$. On the other hand, $\iota$ is a left identity, as it was pointed out before.

We remark that $\iota=1$ if and only if $w$ is the empty word. For example, this is the situation in the $k$-bonacci substitutions.

We say that a semigroup $(\mathcal{S}, *)$ is finitely generated if there exists $\mathscr{G}=\left\{s_{1}, \ldots, s_{m}\right\}$ with $s_{i} \in \mathcal{S}$ such that for all $s \in \mathcal{S}$ we have $s=s_{i_{1}} \star \cdots \star s_{i_{l}}$, for some $l$ and $s_{i_{j}} \in \mathfrak{G}, 1 \leq j \leq l$. The elements of $(\mathfrak{G}$ are called generators of $(\mathcal{S}, \star)$.

We remark that in the Fibonacci case, the set $(\mathscr{P}, \star)$ is not finitely generated because we have the paths $1 e, 1 e e, 1 e e e, \ldots$ that correspond to elements in $\mathscr{P}$ but the word $e$ is not in $\mathscr{P}$. Similarly the semigroup $\left(\mathscr{P}^{+}, \diamond\right)$ is not finitely generated; considering the paths $1 \underbrace{e \cdots e}_{l} 1$, with $l \geq 1$, the numbers associated with these paths cannot be finitely generated since $\epsilon(e)$ is not in $\mathscr{P}^{+}$. The following theorem shows that this is a general situation.

Theorem 3. The semigroups $(\mathscr{P}, \star)$ and $\left(\mathscr{P}^{+}, \diamond\right)$ are not finitely generated.

Proof. We suppose, as before, that $\zeta(1)=1 w 2 w^{\prime}$, where $w=$ $e$ or $w=\underbrace{1 \cdots 1}_{r}$, with $r \geq 1$, and $w^{\prime}$ is a finite word in the alphabet. Let $\alpha:=1 w$ if $w \neq e$ or $\alpha:=1$ if $w=e$. It is a proper prefix of $\zeta(1)$ and $(1, \alpha, 2)$ is a transition in the prefix automaton. Let $a_{l} \cdots a_{1}$ be a path in the prefix automaton such that it starts and ends at the state 1 so that $a_{l} \cdots a_{s}$ does not terminate at the state 1 , for $1<s \leq l$. Let $m$ be the number in $\mathscr{P}^{+}$, such that $\epsilon(m)=a_{l} \cdots a_{1} a_{0}$, with $a_{0}=\alpha$. We consider the numbers $n_{l}=\epsilon(a_{l} \cdots a_{1} \underbrace{e \cdots e}_{l} \alpha)$, with $l \geq 1$; by definition they belong to $\mathscr{P}^{+}$. These numbers cannot be finitely generated, in fact. Let us consider $n_{1}$; if we want to write it as $n_{1}=m \diamond$ $t_{1}$, then $t_{1}$ has to be of the form $\epsilon\left(t_{1}\right)=e \alpha$, but this is not a representation given by the Dumont-Thomas numeration system, since the first symbol is $e$. There is no other possible "factorization" of $n_{1}$, since $\epsilon\left(a_{l} \cdots a_{s}\right) \notin \mathscr{P}^{+}$for $1 \leq s \leq l$. Therefore, $n_{1}$ cannot be written as product of $\mathscr{P}^{+}$, different from itself, and the identities. Similar argument holds for $n_{l}$ for $l>1$. Therefore, $\left(\mathscr{P}^{+}, \diamond\right)$ is not finitely generated.

The proof that $(\mathscr{P}, \star)$ is not finitely generated follows in a similar manner.

We generalize the classical concepts of prime and composite numbers into this context. We say that an element $m$ of $\mathscr{P}^{+}$is $\mathscr{P}^{+}$-composite if there exist $n, n^{\prime} \in \mathscr{P}^{+}$different of an identity element such that $m=n \diamond n^{\prime}$. We say that $m$ is $\mathscr{P}^{+}$-prime if it is not composite. In the proof of Theorem 3 , we have shown that the numbers $n_{l}$ are $\mathscr{P}^{+}$-primes.

Corollary 4. The number $m \in \mathscr{P}^{+}$is $\mathscr{P}^{+}$-prime if and only if $\epsilon(m)=a_{l} \cdots a_{0}$ where $a_{l} \cdots a_{0}$ is a path in the automaton $\mathfrak{A}$ with the property that $a_{l} \cdots a_{s}$ does not terminate at the state 1 for $1<s<l$.

Proof. Let $m \in \mathscr{P}^{+}$whose Dumont-Thomas representation is $\epsilon(m)=a_{l} \cdots a_{0}$ where $a_{l} \cdots a_{0}$ is a path in $\mathfrak{A}$ such that $a_{l} \cdots a_{s}$ does not terminate at the state 1 for $1<s<l$. So the numbers $n_{s}$ given by $\epsilon\left(n_{s}\right)=a_{l} \cdots a_{s}$ are not in $\mathscr{P}$. Since the representation is unique, there are not $n, n^{\prime} \in \mathscr{P}^{+} \backslash\{e\}$ such that $m=n \diamond n^{\prime}$. Hence $m$ is $\mathscr{P}^{+}$-prime.

On the other hand, let $m$ be $\mathscr{P}^{+}$-prime such that $\epsilon(n)=$ $a_{l} \cdots a_{0}$. If there is a $2 \leq r \leq l-1$ such that $a_{l} \cdots a_{r}$ is a path in $\mathfrak{A}$ that terminates at the state 1 , then $m=m_{1} \diamond m_{2}$, where $\epsilon\left(m_{1}\right)=a_{l} \cdots a_{r} \alpha, \epsilon\left(m_{2}\right)=a_{r-1} \cdots a_{0}$, and $(1, \alpha, 2)$ is a transition in the automaton from the state 1 to the state 2 . By construction $m_{1}$ and $m_{2}$ are in $\mathscr{P}^{+}$; therefore, $m$ could not be prime.

We point out that the semigroup $\mathscr{P}^{+}$acts on the set of the positive integers. Let $n \in \mathscr{P}^{+}$, and $m \in \mathbb{N}$; we suppose 
that $m \notin \mathscr{P}^{+}$. If $\epsilon(n)=a_{l} \cdots a_{1} a_{0}$ and $\epsilon(m)=b_{r} \cdots b_{0}$, where $b_{r} \cdots b_{0}$ is a path in the prefix automaton, $\mathfrak{A}$, that terminates at the state $q$, then $a_{l} \cdots a_{1} b_{r} \cdots b_{0}$ is a path in $\mathfrak{A}$, which starts at the initial state and terminates at the state $q$. So $n \diamond m$ is defined and $\epsilon(n \diamond m)=a_{l} \cdots a_{1} b_{r} \cdots b_{0}$; however, $n \diamond m$ is not in $\mathscr{P}^{+}$, if $q \neq 2$. Let $\phi_{n}: \mathbb{N} \rightarrow \mathbb{N}$ be the map defined by $\phi_{n}(m):=n \diamond m$. This map defines a left action of $\mathscr{P}^{+}$on $\mathbb{N}$, since $\phi_{\kappa}$ is the identity, where $\kappa$ is a left identity of $\mathscr{P}^{+}$, and $\phi_{n} \circ \phi_{n^{\prime}}=\phi_{n \diamond n^{\prime}}$.

The dynamics of this action can be studied. Let $m \in \mathbb{N}$; the orbit of $m$ under $\mathscr{P}^{+}$is the set $\left\{\phi_{n}(m): n \in \mathscr{P}^{+}\right\}$, which is denoted by $\mathscr{P}^{+} \diamond m$. Similarly we define the orbit of $m$ under $(\mathscr{P}, \star)$. The following proposition shows how the orbits of both actions are related.

Proposition 5. Let $m$ be a positive integer. Then $\mathscr{P}^{+} \diamond m=$ $(\mathscr{P} \star m) \cup\{m\}$.

Proof. Let $\epsilon(m)=b_{r} \cdots b_{0}$ be the representation of $m$, where $b_{r} \cdots b_{0}$ is a path in the automaton $\mathfrak{A}$ that starts in the initial state and terminates at the state $p$. Let $t \neq m$ be an element of $\mathscr{P}^{+} \diamond m$, so $t=n \diamond m$, for some $n \in \mathscr{P}^{+}$, with $\epsilon(n)=a_{l} \cdots a_{1} a_{0}$, and $l \geq 1$; so $\epsilon(n \diamond m)=a_{l} \cdots a_{1} b_{r} \cdots b_{0}$. Since $a_{l} \cdots a_{1}$ is a path in the automaton that starts and terminates at the initial state, we have $t=n^{\prime} \star m$, where $\epsilon\left(n^{\prime}\right)=a_{l} \cdots a_{1}$. If $t=m$, then $m=\kappa \diamond m$, where $\epsilon(\kappa)=a_{0}$, with $\left(1, a_{0}, 2\right)$ being a transition between the states 1 and 2 . Hence $\mathscr{P}^{+} \diamond m \subset(\mathscr{P} \star m) \cup\{m\}$.

Let $s \in \mathscr{P} \star m$, since $(\mathscr{P}, \star)$ does not have identity $s \neq m$. So $s=n \star m$, for some $n \in \mathscr{P}$. Let $\epsilon(n)=c_{l} \cdots c_{0}$ where $c_{l} \cdots c_{0}$ is a path in the automaton that starts and terminates at the initial state. Hence, $\epsilon(s)=c_{l} \cdots c_{0} b_{r} \cdots b_{0}$. Let $n^{\prime}$ be an element of $\mathscr{P}^{+}$, whose representation is $\epsilon\left(n^{\prime}\right)=c_{l} \cdots c_{0} \alpha$, where $(1, \alpha, 2)$ is a transition from the state 1 to 2 . So $n^{\prime} \diamond m=$ $n$. Therefore, $\mathscr{P} \star m \subset \mathscr{P}^{+} \diamond m$. Clearly $m \in \mathscr{P}^{+} \diamond m$, since $m=\kappa \diamond m$, where $\epsilon(\kappa)=\alpha$.

A further step in the study of the dynamics of this action is the characterization of its invariant sets. The set $Y \subset \mathbb{N}$ is $\left(\mathscr{P}^{+}, \diamond\right)$-invariant if $\cup_{y \in Y} \mathscr{P}^{+} \diamond y=Y$.

\section{Density of the Semigroups}

In this section, we will study the density of the sets $\mathscr{P}$ and $\mathscr{P}^{+}$ in the set of positive integers. Let $\lambda$ be the Perron-Frobenius eigenvalue of $M$ and $v$ and $\omega$ the right and left nonnegative eigenvectors associated with $\lambda$; that is, $M v=\lambda v, \omega^{T} M=$ $\lambda \omega^{T}$. If we choose them so that $\omega^{T} v=\sum_{i=1}^{k} v_{i} \omega_{i}=1$, then the matrix $v \omega^{T}$ is called the Perron projection of $M$ (cf. [20, 21]).

Proposition 6. An estimation of the density of $\mathscr{P}$ in the set of positive integers is given by

$$
\limsup _{N \rightarrow \infty} \frac{1}{N} \#\{m \in \mathscr{P}: m \leq N\} \leq \frac{\omega_{1}}{\omega_{1}+\cdots+\omega_{k}},
$$

where $\omega=\left(\omega_{1}, \ldots, \omega_{k}\right)$ is the left positive eigenvector, associated with Perron-Frobenius eigenvalue, of the transition matrix $M$.
Proof. Let $M_{i j}(n)$ be the $(i, j)$ entry of the matrix $M^{n}$. The number of paths of length $n$ in $\mathfrak{A}$ that start in the state $i$ and end in the state $j$ is given by $M_{i j}(n)$.

Let $R(n)$ be the number of paths of length $n$ in the automaton $\mathfrak{A}$ that start at the initial state; that is, $R(n)=\sum_{j=1}^{k} M_{1 j}(n)$. By the Perron-Frobenius Theorem for primitive matrices $\lim _{n \rightarrow \infty} M^{n} \lambda^{-n}=v w^{T}$ (cf. [21]). So the asymptotical growth of $R(n)$ is given by $\lim _{n \rightarrow \infty} R(n) \lambda^{-n}=\sum_{j=1}^{k}\left(v \omega^{T}\right)_{1 j}$.

Let $P(n)$ be the number of paths of length $n$ in $\mathfrak{A}$, that start in the state 1 and terminate in the state 1 , so $P(n)=M_{11}(n)$. So its asymptotical growth is given by $\lim _{n \rightarrow \infty} P(n) \lambda^{-n}=$ $\left(v \omega^{T}\right)_{11}$. Therefore

$$
\begin{aligned}
& \lim _{N \rightarrow \infty} \sup _{N} \frac{1}{N} \#\{m \in \mathscr{P}: m \leq N\} \\
& \quad \leq \lim _{n \rightarrow \infty} \frac{P(n)}{R(n)}=\lim _{n \rightarrow \infty} \frac{P(n) \lambda^{-n}}{R(n) \lambda^{-n}}=\frac{\left(v \omega^{T}\right)_{11}}{\sum_{j=1}^{k}\left(v \omega^{T}\right)_{1 j}} .
\end{aligned}
$$

This expression simplifies in the following way:

$$
\frac{\left(v \omega^{T}\right)_{11}}{\sum_{j=1}^{k}\left(v \omega^{T}\right)_{1 j}}=\frac{v_{1} \omega_{1}}{\sum_{j=1}^{k} v_{1} \omega_{j}}=\frac{\omega_{1}}{\omega_{1}+\cdots+\omega_{k}} .
$$

We prove in a similar manner the corresponding result for $\mathscr{P}^{+}$.

Corollary 7. An estimation of the density of $\mathscr{P}^{+}$in the set of positive integers is given by

$$
\limsup _{N \rightarrow \infty} \frac{1}{N} \#\left\{m \in \mathscr{P}^{+}: m \leq N\right\} \leq \frac{\omega_{2}}{\omega_{1}+\cdots+\omega_{k}},
$$

where $\omega=\left(\omega_{1}, \ldots, \omega_{k}\right)$ is as in Proposition 6.

Example 8. In the tribonacci case, the transition matrix is

$$
M=\left(\begin{array}{lll}
1 & 1 & 0 \\
1 & 0 & 1 \\
1 & 0 & 0
\end{array}\right)
$$

The Perron-Frobenius eigenvalue is $\lambda$, the real root of the polynomial $x^{3}-x^{2}-x-1$. The left eigenvector of $M$ is $\left(\alpha, \alpha^{2}\right.$, $\left.\alpha^{3}\right)$, where $\alpha=\lambda^{-1}$ and the right eigenvector of $M$ is $\left(\alpha^{2}, \alpha^{3}+\right.$ $\alpha^{4}, \alpha^{3}$ ), without normalization. According to Proposition 6 and Corollary 7, the upper bound of the density of $\mathscr{P}$ is $\alpha \approx$ 0.5436 and that of $\mathscr{P}^{+}$is $\alpha^{2} \approx 0.2955$.

\section{Conflict of Interests}

The author declares that there is no conflict of interests regarding the publication of this paper.

\section{References}

[1] D. E. Knuth, "Fibonacci multiplication," Applied Mathematics Letters, vol. 1, no. 1, pp. 57-60, 1988. 
[2] A. S. Fraenkel, H. Porta, and K. B. Stolarsky, "Some arithmetic semigroups," in Analytic Number Theory: Proceedings of a Conference in Honor of Paul T. Bateman, B. C. Berndt, Ed., pp. 255-264, Birkhauser, Boston, Mass, USA, 1990.

[3] P. Arnoux, "Some remarks about Fibonacci multiplication," Applied Mathematics Letters, vol. 2, no. 4, pp. 319-320, 1989.

[4] V. F. Sirvent, "A semigroup associated with the $k$-bonacci numbers with dynamic interpretation," The Fibonacci Quarterly, vol. 35, no. 4, pp. 335-340, 1997.

[5] A. Messaoudi, "Généralisation de la multiplication de Fibonacci," Mathematica Slovaca, vol. 50, no. 2, pp. 135-148, 2000.

[6] A. Messaoudi, "Tribonacci multiplication," Applied Mathematics Letters, vol. 15, no. 8, pp. 981-985, 2002.

[7] V. F. Sirvent, "Semigroups and the self-similar structure of the flipped Tribonacci substitution," Applied Mathematics Letters, vol. 12, no. 1, pp. 25-29, 1999.

[8] V. Berthé and M. Rigo, Eds., Combinatorics, Automata and Number Theory, vol. 135 of Encyclopedia of Mathematics and Its Applications, Cambridge University Press, Cambridge, UK, 2010.

[9] N. P. Fogg, Substitutions in Dynamics, Arithmetics and Combinatorics, vol. 1794 of Lecture Notes in Mathematics, Edited by V. Berthé, S. Ferenczi, C. Mauduit and A. Siegel, Springer, Berlin, Germany, 2002.

[10] G. Rauzy, "Nombres algébriques et substitutions," Bulletin de la Société Mathématique de France, vol. 110, no. 2, pp. 147-178, 1982.

[11] G. Rauzy, "Sequences defined by iterated morphisms," in Sequences, R. M. Capocelli, Ed., pp. 275-286, Springer, New York, NY, USA, 1990.

[12] S. Eilenberg, Automata, Languages, and Machines, Academic Press, New York, NY, USA, 1974.

[13] J.-M. Dumont and A. Thomas, "Systemes de numeration et fonctions fractales relatifs aux substitutions," Theoretical Computer Science, vol. 65, no. 2, pp. 153-169, 1989.

[14] J.-M. Dumont and A. Thomas, "Digital sum moments and substitutions," Acta Arithmetica, vol. 64, no. 3, pp. 205-225, 1993.

[15] J.-P. Allouche and J. Shallit, Automatic Sequences, Cambridge University Press, Cambridge, UK, 2003.

[16] V. Berthé and M. Rigo, "Abstract numeration systems and tilings," in Proceedings of the 32nd Symposium, Mathematical Foundations of Computer Science, vol. 3618 of Lecture Notes in Computer Science, pp. 131-143, Springer, 2007.

[17] C. Frougny, "Representations of numbers and finite automata," Mathematical Systems Theory, vol. 25, no. 1, pp. 37-60, 1992.

[18] P. J. Grabner, P. Liardet, and R. F. Tichy, "Odometers and systems of numeration,” Acta Arithmetica, vol. 70, no. 2, pp. 103-123, 1995.

[19] N. Bourbaki, Elements of Mathematics: Algebra I, Springer, Berlin, Germany, 2007.

[20] F. Gantmacher, The Theory of Matrices, vol. 2, American Mathematical Society, Providence, RI, USA, 2000.

[21] R. A. Horn and C. R. Johnson, Matrix Analysis, Cambridge University Press, Cambridge, UK, 1985. 


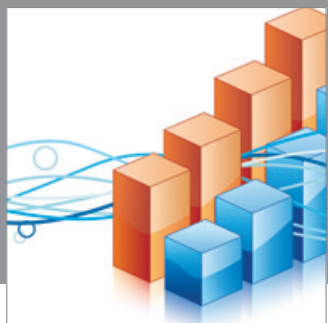

Advances in

Operations Research

mansans

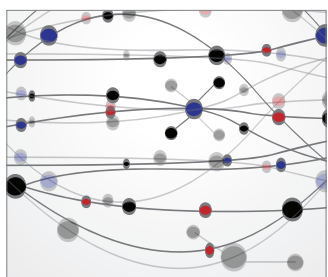

The Scientific World Journal
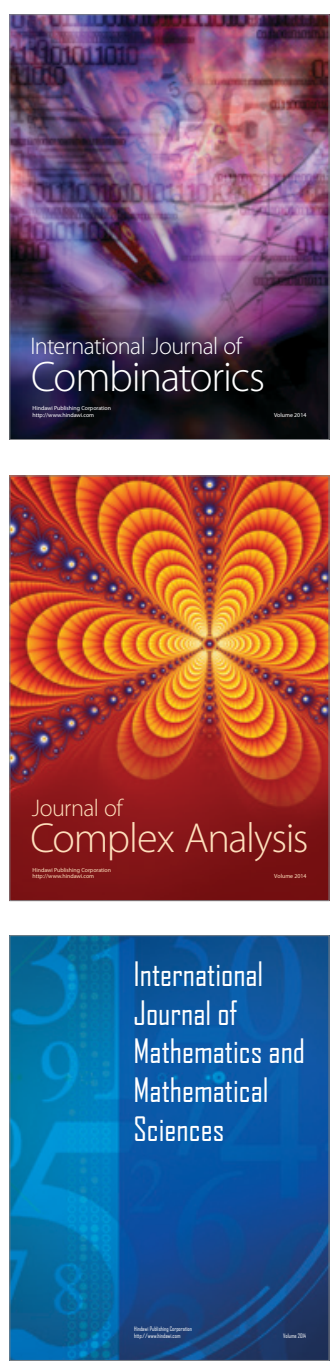
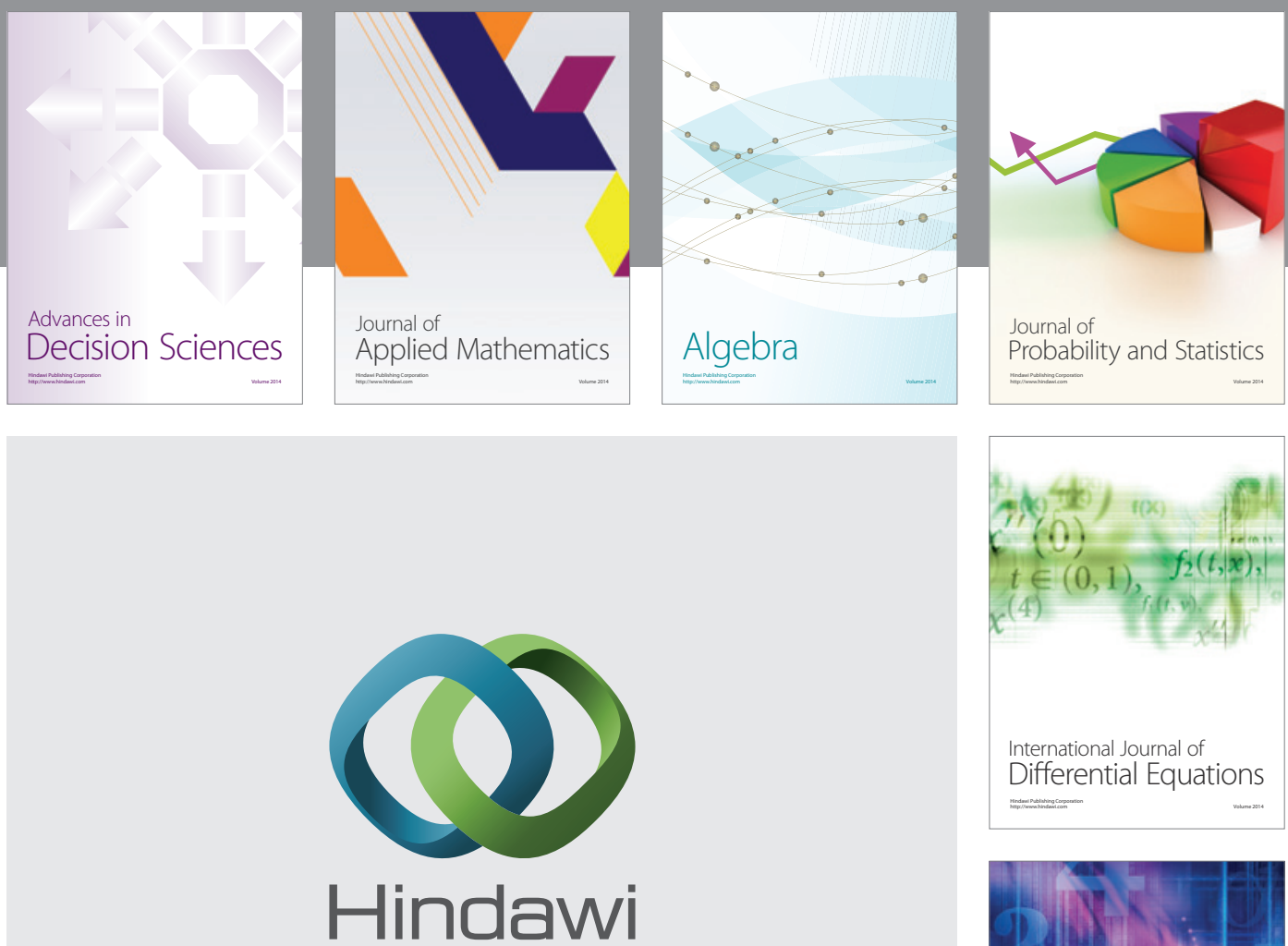

Submit your manuscripts at http://www.hindawi.com
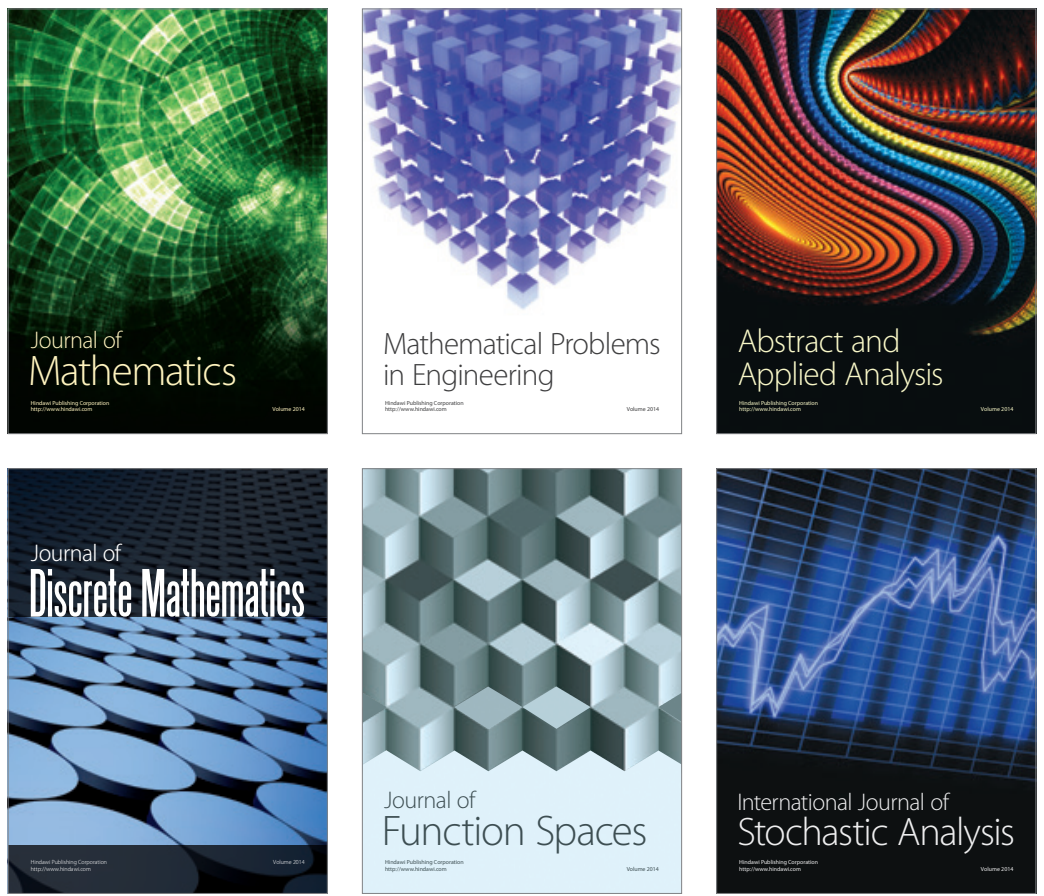

Journal of

Function Spaces

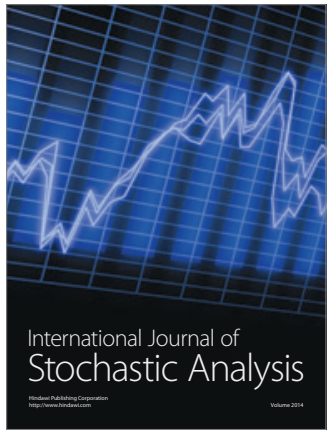

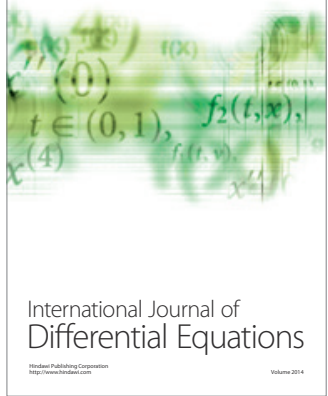
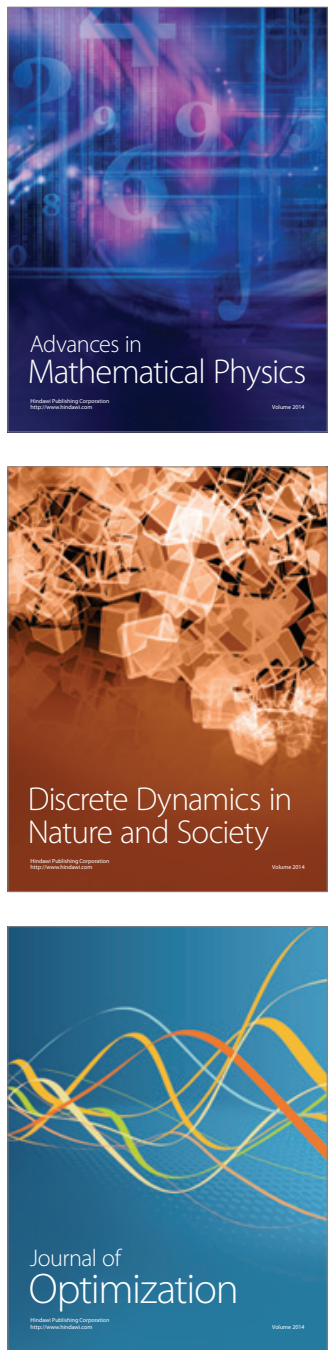